\title{
CONTRIBUTION OF SMALL-SCALE CORRELATED FLUCTUATIONS OF MICROSTRUCTURAL PROPERTIES OF A SPATIALLY EXTENDED GEOPHYSICAL TARGET UNDER THE ASSESSMENT OF RADAR BACKSCATTER
}

\author{
B.S. Yurchak \\ University of Maryland, Baltimore County, GEST, MD 20771, USA-yurboris@umbc.edu
}

\begin{abstract}
The study of the collective effects of radar scattering from an aggregation of discrete scatterers randomly distributed in a space is important for better understanding the origin of the backscatter from spatially extended geophysical targets (SEGT). We consider the microstructure irregularities of a SEGT as the essential factor that affect radar backscatter. To evaluate their contribution this study uses the "slice" approach: particles close to the front of incident radar wave are considered to reflect incident electromagnetic wave coherently. The radar equation for a SEGT is derived. The equation includes contributions to the total backscatter from correlated small-scale fluctuations of the slice's reflectivity. The correlation contribution changes in accordance with an earlier proposed idea by Smith (1964) based on physical consideration. The slice approach applied allows parameterizing the features of the SEGT's inhomogeneities.
\end{abstract}

Index Terms- Remote sensing, radar scattering

\section{INTRODUCTION}

The study of the collective effects of radar scattering from an aggregation of discrete scatterers randomly distributed in a space is important for better understanding the origin of the backscatter deviations from the theoretical models (e.g., $[1,2])$. In the current paper we analyze a mechanism which can cause the backscatter to deviate from the classical (incoherent) estimate because of the collective effects in spatially extended geophysical targets (SEGT). Description of this mechanism is based on so-called "slice" approach firstly suggested for the meteorological SEGT (clouds, rain) in $[3,4]$, and enhanced by the author [5] for general SEGT (including thick snow cover), taking into account the statistics of its scattering properties. The approach exploits the partial coherence of the backscatter electric field from particles located close to the wavefront of the incident radar irradiance within a radial distance $\left(\Delta_{s}\right)$ that is much less than the radar wavelength $(\lambda)$. This fictitious thin volume is a "slice", Fig. 1.

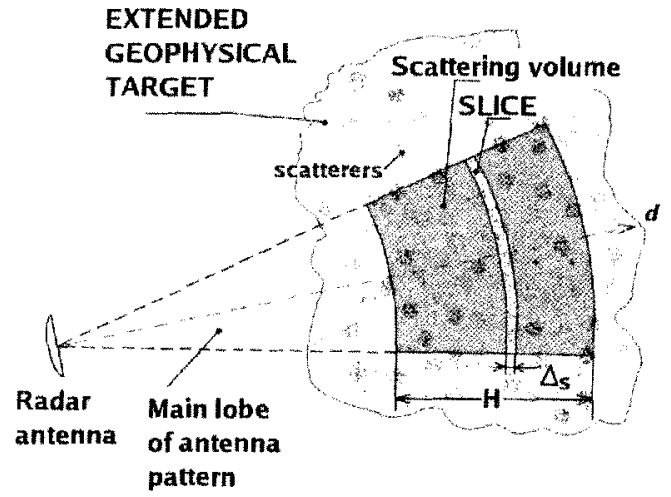

Fig.1. Simplified slice approach scheme. An arbitrary position of a single slice is shown. The scattering volume is shaded

The corresponded radar equation contains parameters that parameterize the irregularities of the SEGT's microstructure. Here we extend that parameterization with a correlative factor that describes the correlation between slices' reflectivity and, in particular, interprets an increase or decrease in the backscatter compared to the expected one for some cases.

\section{RADAR CROSS SECTION OF THE SEGT IN A "SLICE" APPROACH}

The mean radar cross section (MRCS) of the volume' component of the backscatter from SEGT under assumption of single scattering by individual particles has the follow general form:

$$
\left\langle\sigma_{\Sigma}\right\rangle=\left\langle\left|\sum_{i=1}^{N} a_{i} \exp \left(-j 2 k d_{i}\right)\right|^{2}\right\rangle
$$

where $a \equiv \sqrt{\sigma}$ is the "Particle Radar Equivalent Length" (PREL), $\sigma$ is the random RCS of an individual particle, $N$ is the total number of particles within the scattering volume, $k=2 \pi \lambda^{-1}$ is the wavenumber of the incidence radar 
irradiance of the wavelength $\lambda$. Under a "slice" approach the instantaneous sum of PRELS of particles located close to the wavefront at the $q$ th position and within the slice radial size $\Delta_{s} \ll<\lambda$ can be represented as:

$$
\sum_{i \in\{q\}} a_{i}^{(q)} \exp \left(-j 2 k d_{i}^{(q)}\right)=b_{q} \exp \left(-j 2 k d_{q}\right)
$$

where $b_{q}=\sum_{i \in\{q\}} a_{i}^{(q)}=\sum_{i \in\{q\}}^{n_{q}} a_{i}^{(q)} \quad$ is the "Slice Radar

Equivalent Length" (SREL) which is the sum of all PRELs within a slice; $n_{q}$ is a random number of particles inside a slice, and $d_{q}=q \Delta_{\mathrm{s}}$ is a distance of $q$ th slice from the border of the scattering volume nearest to the radar. Thus, the backscattering feature of the radar scattering volume can be represented as the series of $M=H / \Delta_{s} \gg 1$ adjoining "pulses" (slices) with random amplitude (b):

$$
\begin{aligned}
& \left\langle\sigma_{\Sigma}\right\rangle=\left\langle\left|\sum_{q=1}^{M} b_{q} \exp \left(-j 2 k q \Delta_{s}\right)\right|^{2}\right\rangle= \\
& =\left\langle\left|\frac{1}{\Delta_{s}} \int_{-H / 2}^{H / 2} b(x) \exp (-j 2 k x) d x\right|^{2}\right\rangle
\end{aligned}
$$

The spatial distribution of the SREL along the radial direction $b(\mathrm{x})$ within the scattering volume can be represented as a sum of a quasi-regular component $b_{0}(\mathrm{x})$ and a small-scale (compared with the wavelength) fluctuating component $b_{f}(\mathrm{x})[6]$ :

$$
b(x)=b_{0}(x)+b_{f}(x)
$$

If assume that the process $b(x)$ is stationary, i.e., $\left(b_{j}(x)\right)=0, \int_{-\pi / 2}^{2} b_{0}(x) \exp (-j 2 k x) d x \approx 0$,

and

$\operatorname{Var}(b)=$ const, it is possible to show [5] that MRCS can be expressed through the normalized correlation function $R(\zeta)$ of the fluctuating component of SREL:

$$
\left\langle\sigma_{\Sigma}\right\rangle=\frac{\operatorname{Var}(b)}{\Delta_{s}^{2}} \int_{-H / 2}^{H / 2} d x \int_{-H / 2}^{-H / 2} d y \cdot R(x-y) \exp [-j 2 k(x-y)]
$$

where $\operatorname{Var}(b)=\operatorname{Var}(a) \cdot\langle n\rangle+\langle a\rangle^{2} \cdot \operatorname{Var}(n) \quad$ is the variation of SREL, expressing through the statistics of PREL and particle number within a slice basing on the known theorem about the variance of a sum of a variable number of variable values [7].

In the case of uncorrelated fluctuations of SREL, i.e., when

$$
R(x-y)=\left\{\begin{array}{l}
1, x-y=0 \\
0, x-y \neq 0
\end{array}\right.
$$

it was shown by the author [5] that the MRCS is equal to:

$$
\left\langle\sigma_{\Sigma}\right\rangle=\left\langle\sigma_{\Sigma}\right\rangle_{\text {ctass }} \operatorname{dev} F\left(\xi_{a}, \chi\right)
$$

where $\left\langle\sigma_{\Sigma}\right\rangle_{\text {cluss }}$ is the MRCS in the "classical" (incoherent) approach; and

$$
\operatorname{dev} F\left(\xi_{a}, \chi\right)=\frac{\xi_{a}^{2}+\chi}{\xi_{a}^{2}+1}
$$

is the deviation factor, governed by the Poisson index $\chi=\frac{\operatorname{Var}(n)}{\langle n\rangle},(n$ is a random number of particles within a slice), and by the variation coefficient of PREL $\xi_{a}=\frac{\operatorname{Stdev}(a)}{\langle a\rangle}$. If the fluctuations of particle number is pertaining to the Poisson law when $\chi=1$, then for any $\xi_{a}$ the deviation factor $\operatorname{dev} F=1$, and a classical result of incoherent approach takes place. The PREL variation coefficient $\left(\xi_{a}\right)$ can be expressed through the parameters of the particle size distribution function (PSDF) [5]:

$$
\xi_{a}=\xi_{r}^{-1 / 3} \frac{\sqrt{\xi_{r}^{-1}+2 S k}}{\xi_{r}^{-3}+3 \xi_{r}^{-1}+S k}
$$

where $\xi_{r}=\frac{\operatorname{Stdev}(r)}{\langle r\rangle}$ is the particle size variation coefficient, and $S k$ is the skewness coefficient.

Although the deviation factor (8) describes the plus/minus deviations of the MRCS from the classical one, there is also one more additional slice's statistics that can contributes in the backscatter as well.

\section{MEAN RCS TAKING INTO ACCOUNT THE CORRELATION BETWEEN SLICE SCATTERING PROPERTIES}

In a general case the SRELs can be correlated due to finite $b$-disturbances spectrum with inner $\left(l_{0}\right)$ and outer $\left(L_{0}\right)$ scales. The slice size is assumed to be equal to the minimal scale of SREL's fluctuations $l_{0}$ (if $l_{0}<\lambda$ ). In this case equation (5) can be written in the form:

$$
\left\langle\sigma_{\Sigma}\right\rangle=\frac{\operatorname{Var}(b)}{\Delta_{s}^{2}} H \int_{H / 2}^{H / 2} d \Delta\left(1-\frac{|\Delta|}{H}\right) R(\Delta) \exp (-j 2 k \Delta)
$$

If assume that the correlation interval is much less than the radial size of the scattering volume, $(10)$ can be reduced to:

$$
\begin{aligned}
& \left\langle\sigma_{\Sigma}\right\rangle \approx \frac{\operatorname{Var}(b)}{\Delta_{s}^{2}} H \int_{-H / 2}^{\pi / 2} d \Delta R(\Delta) \exp (-j 2 k \Delta)= \\
& =M \cdot \operatorname{Var}(b) \cdot \operatorname{corr} F
\end{aligned}
$$

where $\operatorname{corr} F=\frac{1}{\Delta_{s}} F(\kappa)_{\kappa=2 k}$ is the correlative factor of the normalized spectral function $F(\kappa)$. Since

$$
\begin{aligned}
& M \cdot \operatorname{Var}(b)=M\langle n\rangle\langle a\rangle^{2}\left(\xi_{a}^{2}+\chi\right)= \\
& =N\langle a\rangle^{2} \cdot \operatorname{dev} F \cdot\left(\xi_{u}^{2}+1\right)=\left\langle\sigma_{\Sigma}\right\rangle_{\text {tatas }} \operatorname{dev} F
\end{aligned}
$$

the equation (11) can be represented in the form:

$$
\left\langle\sigma_{\Sigma}\right\rangle=\left\langle\sigma_{\Sigma}\right\rangle_{\text {ctass }} \cdot \operatorname{dev} F \cdot \operatorname{corr} F
$$

Spectral function for process (3) is [8]: 


$$
F(\kappa)=\Delta_{s}^{-1}\left|F_{s}\left(\kappa, \Delta_{s}\right)\right|_{q=-\infty}^{2} \sum_{q=\infty}^{=\infty} R\left(q \Delta_{s}\right) \exp \left(j k q \Delta_{s}\right)
$$

where $F_{s}\left(\kappa, \Delta_{s}\right)$ is the normalized spectral function of the typical "pulse" of SREL.

Assuming the radial distribution of SREL within the scattering volume as a consequence of adjoining rectangular "pulses" of width $\Delta_{\mathrm{s}}$ with the exponential correlation function $R(\Delta)=\exp \left(-\left|\Delta \cdot \Delta_{0}^{-1}\right|\right)$, the following expression can be derived for the correlative factor:

$$
\operatorname{cor} F=\left|\frac{\sin \left(k \Delta_{s}\right)}{k \Delta_{s}}\right|^{2} \frac{1-\exp \left(-2 \frac{\Delta_{x}}{\Delta_{i n}}\right)}{1-2 \exp \left(-\frac{\Delta_{s}}{\Delta_{0}}\right) \cdot \cos \left(2 k \Delta_{s}\right)+\exp \left(-2 \frac{\Delta_{s}}{\Delta_{0}}\right)}
$$

The plot of (14) is depicted in Fig.2 for different ratios of $\left(\Delta_{\mathrm{q}} / \Delta_{\mathrm{s}}\right)$.

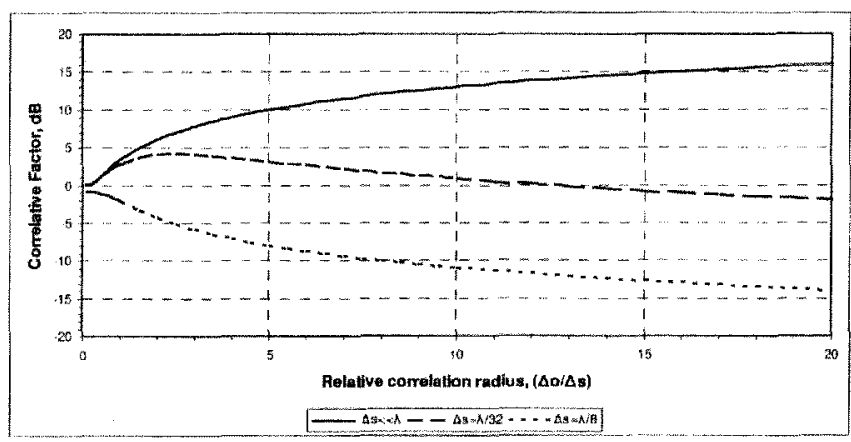

Fig.2. Correlative factor versus relative correlation radius $\left(\Delta_{0} / \Delta_{\varsigma}\right)$ for different slice sizes.

As follows from this plot the correlative factor for thin slices $\left(\Delta_{s}<\lambda / 16\right)$ and a small correlation radius arises because of the small differences in the distance between slices, which cause a constructive interference. Since in the case of atmospheric turbulence, $\Delta_{s}=l_{0} \approx \sqrt[4]{v^{3} / \varepsilon}$ ( $v$ is the kinematic viscosity of air, and $\varepsilon$ is the energy dissipation per unit of mass), this case corresponds to the moderate and high turbulence. The upper curve is only a theoretical limit that never can be reached in practice because it relates to the infinitesimal scales of fluctuations, which cannot be less than the inner scale $\left(l_{0}\right)$ of SREL's disturbance.

Subsequent increases in the correlation interval causes the contribution of the destructive interference, which makes the backscatter decrease. P.L. Smith [4] predicted qualitatively this behavior based on the interference theory. With a slice size of $\sim \lambda / 8$ suggested in [6] (low turbulence), the correlative factor slows monotonously beginning at $0 \mathrm{~dB} ; \operatorname{Corr} F \approx-10$ $\mathrm{dB}$ at $\Delta_{0} \approx \lambda$, for example. This value is close to the observable deviation of the backscatter from the atmospheric fog [6]. The arising feature of the correlative factor together with the probably high value of the Poisson index [9] can be applied for interpretation of the experimental data obtained during the radar probing of clouds accompanied with simultaneous measurements of the particle size spectra in situ [10]. In this experiment, the estimations of radar reflectivity based on the standard weather equation were found notably higher than expected values calculated according to the spectrometer data.

\section{CONCLUDING REMARKS}

The radar backscatter features have been considered within the frames of the slice approach taking into account the correlation between slice radar equivalent lengths. The correlation contribution has been evaluated based on the derived correlative factor. In particular, for a random scattering medium with correlation radius less than the wavelength this factor describes the backscatter changes in accordance with an earlier proposed idea based on physical consideration [4]. The slice approach allows interpreting the variety of radar backscatter deviations from the classical model based on the inventory of contribution of the statistical features of the fine-scale microstructural fluctuations. The statistics of a slice radar equivalent length (SREL), commonly unknown for spatially extended geophysical targets (SEGT), should be investigated in future researches. The correlative factor obtained together with the deviation factor [5] parameterizes the "rate of inhomogeneity" of a SEGT. The "classical" result (the total RCS is a sum of the RCSs of individual particles) takes place upon 2 conditions: (1) the fluctuations of particle number at small-scale should be pertaining to the Poisson law, and (2) SREL fluctuations should be not correlated. The assessment of these factors and conditions inherent to different kinds of a SEGT will improve the accuracy and reliability of the radar remote sensing.

\section{AKNOWLEDGEMENT}

This work was supported by NASA's Cryospheric Science Program

\section{REFERENCES}

[1] C.R. Noveltis, "Feasibility study of imaging the Antarctic ice using a spaceborne P-band radar," Electromagnetic Model Review Document, 2005. ESA Contract Number 18195/04/NL/CB. WP 200.

[2] J.R. Kendra, K. Sarabandi, and F.T. Ulaby, "Radar measurements of snow: experiment and analysis," IEEE Trans. Geosci. Remote Sens., vol. 36, no. 3, pp. 864-879. May 1998. 
[3] J.S. Marshal and W. Hitschfeld, "Interpretation of the fluctuating echo from randomly distributed scatterers," Can. J. Phys., vol. 31, pt. I, pp. 962-994, 1953.

[4] P.L. Smith, Jr., "Scattering of microwave by cloud droplets," in Proc. $11^{\text {th }}$ Weather Radar Conf., Sep. 14-18, 1964, pp. 202-207.

[5] B.S. Yurchak, "Radar volume backscatter from spatially extended geophysical targets in a "slice" approach, IEEE Trans. Geosci. Remote Sens., vol.47, no 11, pp. 3690-3696, November 2009.

[6] K. Naito, and D. Atlas, "On microwave scatter by partially coherent clouds," in Proc. $12^{\text {th }}$ Weather Radar Conf., Oct. 17-20, 1966, pp. 7-12.

[7] A.W. Drake, Fundamentals of Applied Probability Theory. McGraw-Hill, New York, 1967.

[8] V.T. Goryainov, A.G. Zhuravlev, and V.I. Tikhonov. Staistical Radio Engineering: Examples and problems. Textbook, Sov. Radio, Moscow, 1980, 544 pp.

[9] A. B. Kostinski and A. R. Jameson, "On the spatial distribution of cloud particles," J. Atmos. Sci., vol. 57, no. 7, pp. 901-915, Apr. 2000.

[10] F. Pasqualucci, N.L. Abshire, R.B. Chadwick, and R.A. Kropfli, "Cloud observations during the Phoenix experiment," in Proc. $19^{\text {th }}$ Conf. Radar Meteorol., April 1518,1980 , pp. $715-117$. 


\section{CONTRIBUTION OF SMALL-SCALE CORRELATED FLUCTUATIONS OF MICROSTRUCTURAL. PROPERTIES OF A SPATIALLY EXTENDED GEOPHYSICAL TARGET UNDER THE ASSESSMENT OF RADAR BACKSCATTER}

\section{B.S. Yurchak}

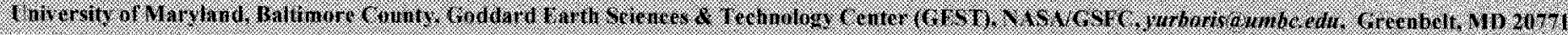
Abstract The study of the collective effects of radar scattering from an aggregation of discrete scatterers randomly distributed in a space is important for better understanding the origin of the back scatter deviations from spatially, extended geophysical targets (SEGT). We consider the microstructure irregularities of a SEGT as the essential factor that affect radar backscatter. To evaluate the ir contribution electromagnetic wave coherently. Each slice is much narrower than the radar wavelength in the wave propagation direction. The radar equation for a $S E G T$, which is based on the slice model backscatter volume, is derived. The equation includes contributions to the total backscatter from correlated small-scale fluctuations of the slice's reflectivity The average power of a slice model of proportional to the sixth moment of the particles size distribution function (classical case), only for the case of Poisson fluctuations of particle concentration within the slices and uncorrelated small-scale reflectivity fluctuations within a back satter volume. The correlation contribution changes in accordance with an earlier proposed idea by Smith (1964) based on physical consideration. The slice approach
applied allows parameterizing the features of the $S E G T$ s inhomogeneities.

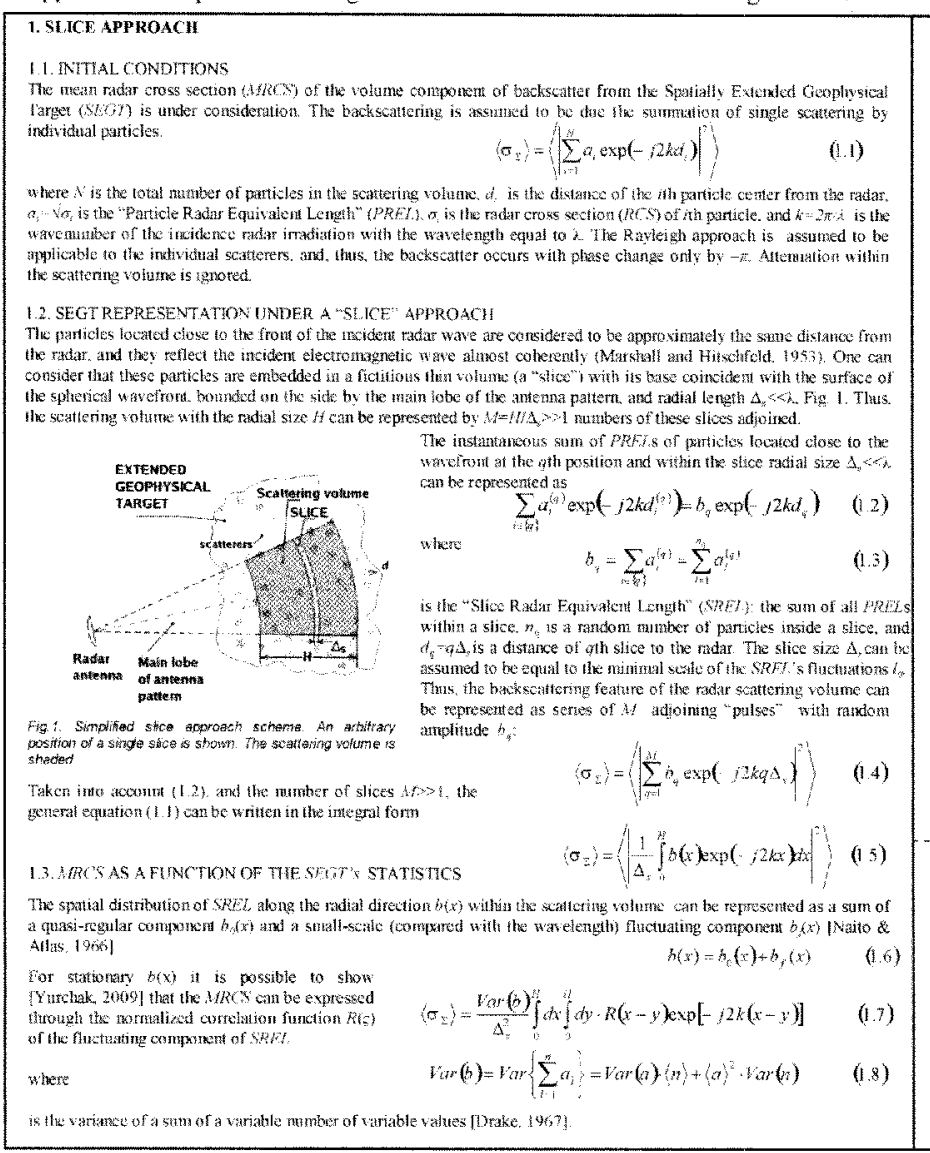

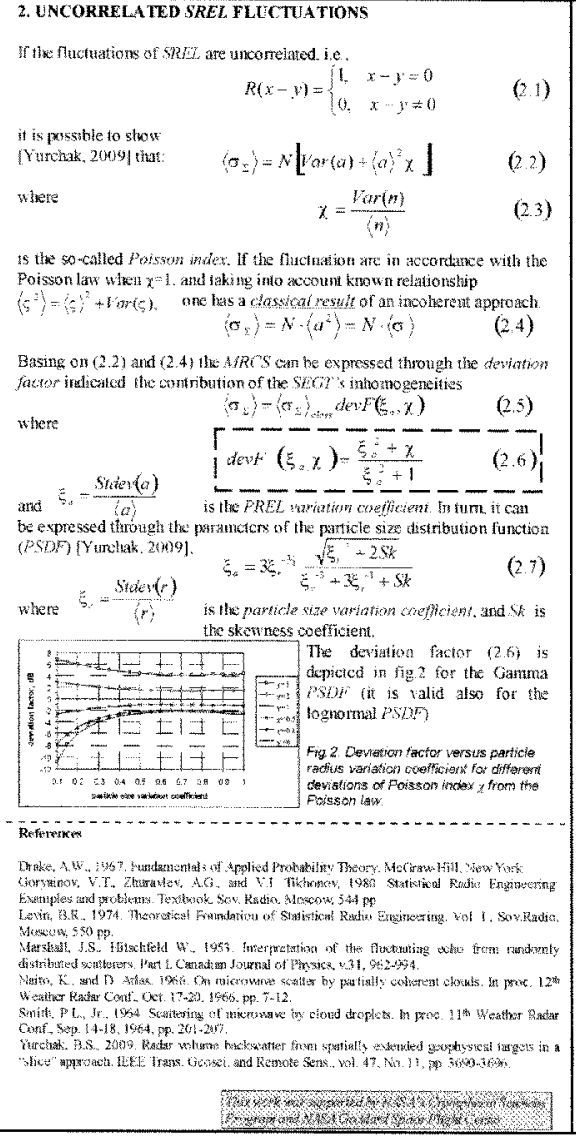

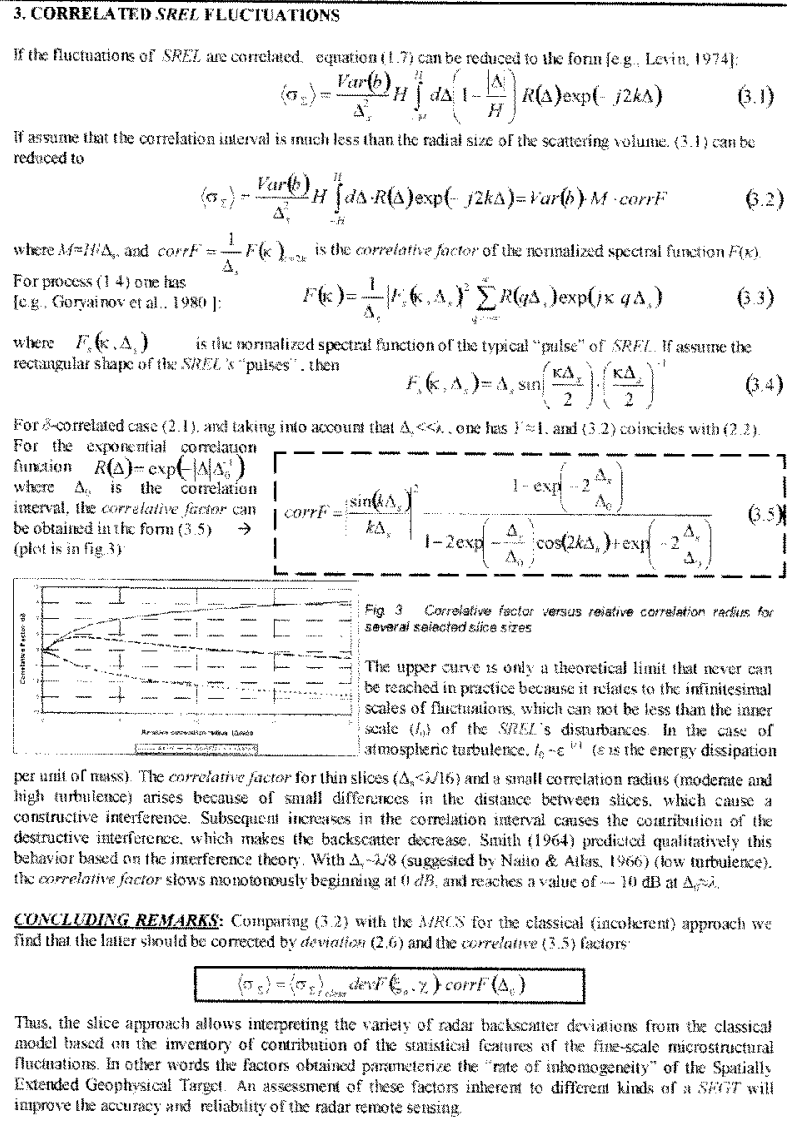

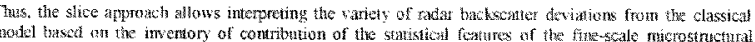

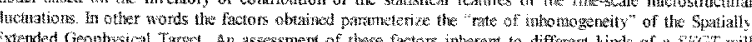

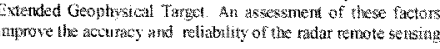

